\title{
Методический подход к оценке эффективности инжиниринговых проектов внедрения киберфизических систем в деятельность промышленных предприятий
}

\author{
Любименко Д.А., Вайсман Е.Д. \\ Южно-Уральский государственный университет (НИУ), \\ Россия, 454080, г. Челябинск, проспект Ленина, 76 \\ E-mail: lyubimenko.da@mail.ru, vaismaned@susu.ru
}

\begin{abstract}
Аннотация. В современных условиях цифровизация, являясь драйвером прогресса, влияет на все сферы в обществе, в том числе и на экономику. В связи с этим особый интерес представляют собой инжиниринговые цифровые проекты, к которым относят киберфизические системы. Несмотря на активное внедрение в сфере промышленности, такие проекты малоизучены. Целью данного исследования является разработка методического подхода к оценке эффективности киберфизических систем. В результате исследования проблемы были проанализированы различные виды киберфизических систем, определены их особенности и выполнена классификация. С учетом выявленной специфики проектов предложен методический подход к оценке их эффективности с помощью показателя ОЕЕ. Результаты исследования открывают новое теоретическое направление в исследовании проблемы цифровых инвестиционных проектов.
\end{abstract}

Ключевые слова: цифровизация, инжиниринг, киберфизические системы, OEE, методика UNIDO, доступность, производительность, качество, эффективность работы оператора.

Для цитирования: Любименко Д.А., Вайсман Е.Д. 2021. Методический подход к оценке эффективности инжиниринговых проектов внедрения киберфизических систем в деятельность промышленных предприятий. Экономика. Информатика. 48(4): 663-678. DOI: 10.52575/2687-09322021-48-4-663-678.

\section{Methodological approach to assessing the effectiveness of engineering projects for the implementation of cyber-physical systems in the activities of industrial enterprises}

\author{
Darya A. Lyubimenko, Elena D. Vaisman \\ South Ural State University (National Research University) \\ 76 Lenin prospekt, Chelyabinsk, Russia, 454080 \\ E-mail: lyubimenko.da@mail.ru, vaismaned@susu.ru
}

\begin{abstract}
In modern conditions, digitalization, being a driver of progress, affects all spheres in society, including the economy. In this regard, engineering digital projects, which include cyber-physical systems, are of particular interest. They combine software and hardware. Even though such projects are being actively implemented in industry, they are poorly studied, their implementation requires significant resources, and there is no single approach to the analysis of their effectiveness. In this regard, the issue that became the goal of this study is relevant - the development of a methodological approach to assessing the effectiveness of cyber-physical systems. As a result of the study of the problem, various types of cyber-physical systems were analyzed, their features were determined, and a classification was made. The existing methods for assessing the effectiveness of digital investment projects are considered, none of which considers the specifics of cyber-physical systems. A unified methodological approach to assessing their effectiveness using the OEE indicator is proposed. The results of the study open a new theoretical direction in the study of the problem of digital investment projects.
\end{abstract}


Keywords: digitalization, engineering, cyber-physical system, OEE, UNIDO method, availability, productivity, quality, operator efficiency.

For citation: Lyubimenko D.A, Vaisman E.D. 2021. Methodological approach to assessing the effectiveness of engineering projects for the implementation of cyber-physical systems in the activities of industrial enterprises. Economics. Information technologies. 48(4): 663-678 (in Russian). DOI: 10.52575/2687-09322021-48-4-663-678.

\section{Введение}

Цифровизация коренным образом меняет жизнь человека во всех сферах. Она является драйвером прогресса, который развивается все более стремительно. Сегодня скорость проникновения новых технологий в человеческую жизнь стала феноменальной. Например, в 1992 г. была отправлена первая смс, а к 2015 г. ежесекундно в мире отправлялись двадцать тысяч сообщений [Круглов]. Аналогичная тенденция наблюдается не только в сфере программного обеспечения (неосязаемых технологий), но касается также физических устройств. Так, в январе 2007 года на рынке появился первый iPhone, а к 2016 г. обладателем телефона Apple стал уже каждый восьмой житель планеты [Архангельская и др.].

Существует мнение, что выпуск іPhone был технологическим прорывом. Однако это не совсем так. Создание первого телефона корпорации Apple является скорее инжинирингом. Термин характеризует разработку новых продуктов, в которой применяются как уже имеющиеся, так и новые технологии. Стив Джобс в іPhone объединил сенсорный дисплей, Интернет, GPS, протоколы TCP/IP, которые уже существовали до него. Искусство инжиниринга при этом заключается в умении разработчика удовлетворить потенциальные потребности рынка, которые еще даже не известны потребителям, и создать качественный продукт с высокой добавленной стоимостью. При этом его основой является существующая технологическая база.

В условиях производственного предприятия инжиниринг приобретает иные масштабы и из продуктового превращается в промышленный. Он предполагает создание производственных цехов, заводов и соответствующей инфраструктуры. Усложнение технологий и приобретение создаваемыми объектами комплексного характера стимулировало проминжиниринг развиваться и расширять сферу своего влияния. На сегодняшний день он подразумевает не только создание концепции и проекта цеха, фабрики или завода, но и сопровождение реализуемого цифрового инвестиционного проекта. В промышленности искусство инжиниринга заключается в способности осуществить модернизацию производства любым доступным способом.

Например, компания решает выпускать новый продукт, установив современное оборудование. В этом случае денежный поток будет направлен на покупку оборудования, реконструкцию производственной площадки и формирование необходимых инженерных коммуникаций. Прежде всего следует произвести аудит всех процессов на предприятии, чтобы определить, как новая технология будет реализована в условиях уже существующей системы. После этого формируется бюджет на внедрение оборудования. На этом этапе зачастую становится очевидно: финансирование проекта не соответствует возможностям компании. Наиболее простым решением было бы отказаться от модернизации, но с точки зрения инжиниринга выгоднее взять за основу уже имеющееся оборудование и на нем реализовывать новую технологию. Таким образом, переналадка производственных линий позволит выпускать новый продукт, избежав дополнительных капитальных вложений.

Этот подход широко используется при реализации отдельных цифровых решений в промышленности. Комплексная модернизация производства в связи с высокой стоимостью недоступна многим предприятиям, но преобразования, предполагающие установку новых программных продуктов или устройств на имеющееся оборудование, уже идут. Описанные процессы свидетельствуют о приближении к концепции «Индустрия 4.0». Интернет вещей, 
искусственный интеллект, Big Data, нейронные сети - все это уже применяется компаниями в своей деятельности. Технологии пока разрозненны, хоть и демонстрируют отличные результаты. По этой причине пока преждевременно говорить о становлении того типа промышленности, который предполагает концепция «Индустрия 4.0» [Клейменова, 2021].

Однако, чтобы успешно преодолеть путь к ней, требуется инжиниринг, объединяющий существующие технологические и цифровые решения в единый комплекс киберфизическую систему. Она представляет собой особый вид цифровых инвестиционных проектов.

\section{Формирование методического подхода к оценке эффективности киберфизических систем}

Термин «киберфизическая система» был введен в 2006 году Хелен Гилл из Национального научного фонда США для обозначения интеграции вычислений с физическими процессами и произошел от слова «кибернетика», который, в свою очередь, своим появлением обязан Норберту Винеру, американскому математику, оказавшему огромное влияние на развитие теории систем управления [Lee, Seshia, 2017]. Во время Второй мировой войны Винер изобрел и применил на практике технологию автоматического прицеливания и стрельбы из зенитных орудий. В ней были реализованы принципы аналогичные тем, которые сегодня используются в компьютерных системах управления с обратной связью. Винер считал, что кибернетика объединяет контроль и коммуникации [Lee, Seshia, 2017].

На сегодняшний день исследователи пока не пришли к единому мнению в вопросе определения термина киберфизической системы (cyber-physical system, CPS). По мнению исследовательской группы киберфизических систем Мельбурнского королевского технологического института под руководством профессора Роберто Сабатини, киберфизическая система объединяет из различных научных теорий и инженерных дисциплин, в том числе, кибернетики, встроенные системы, распределенное управление, сенсорные сети, теорию управления и системную инженерию. Благодаря интеграции физических и цифровых компонентов максимизируется синергетический эффект, что позволяет добиться лучших результатов с точки зрения производительности и стоимости конечного продукта. Роберто Сабатини также выделяет три фундаментальных компонента, при которых возможно функционирование CPS: связь, управление, вычисление [What are cyber-physical systems?].

В наиболее общем случае под киберфизической системой понимают комплексную систему вычислительных и физических элементов, получающих данные из внешней среды и применяющих их в целях оптимизации производственных и управленческих процессов [Руководство по цифровой трансформации производственных предприятий, 2019]. CPS объединяет программное обеспечение и аппаратное обеспечение (датчики, процессоры, коммуникативные технологии) и способна автономно обмениваться информацией с другими системами и выполнять определенные функции. Она подразумевает взаимодействие с человеком или работает самостоятельно [Cyber-Phisical System. Driving force for innovation in mobility, healthy, energy and production, 2011].

В других источниках киберфизическую систему рассматривают как информационнотехнологическую концепцию, которая объединила кибернетику, механотронику, теорию и практику управления процессами [Трошин, Пискайкин, 2021]. Преимущество CPS заключается в возможности принимать более точные и объективные решения, минимизировав влияние человеческого фактора.

Рассмотрим классификацию киберфизических систем (табл. 1). 
Классификация киберфизических систем (КФС)

The cyber-physical systems classification (CPS)

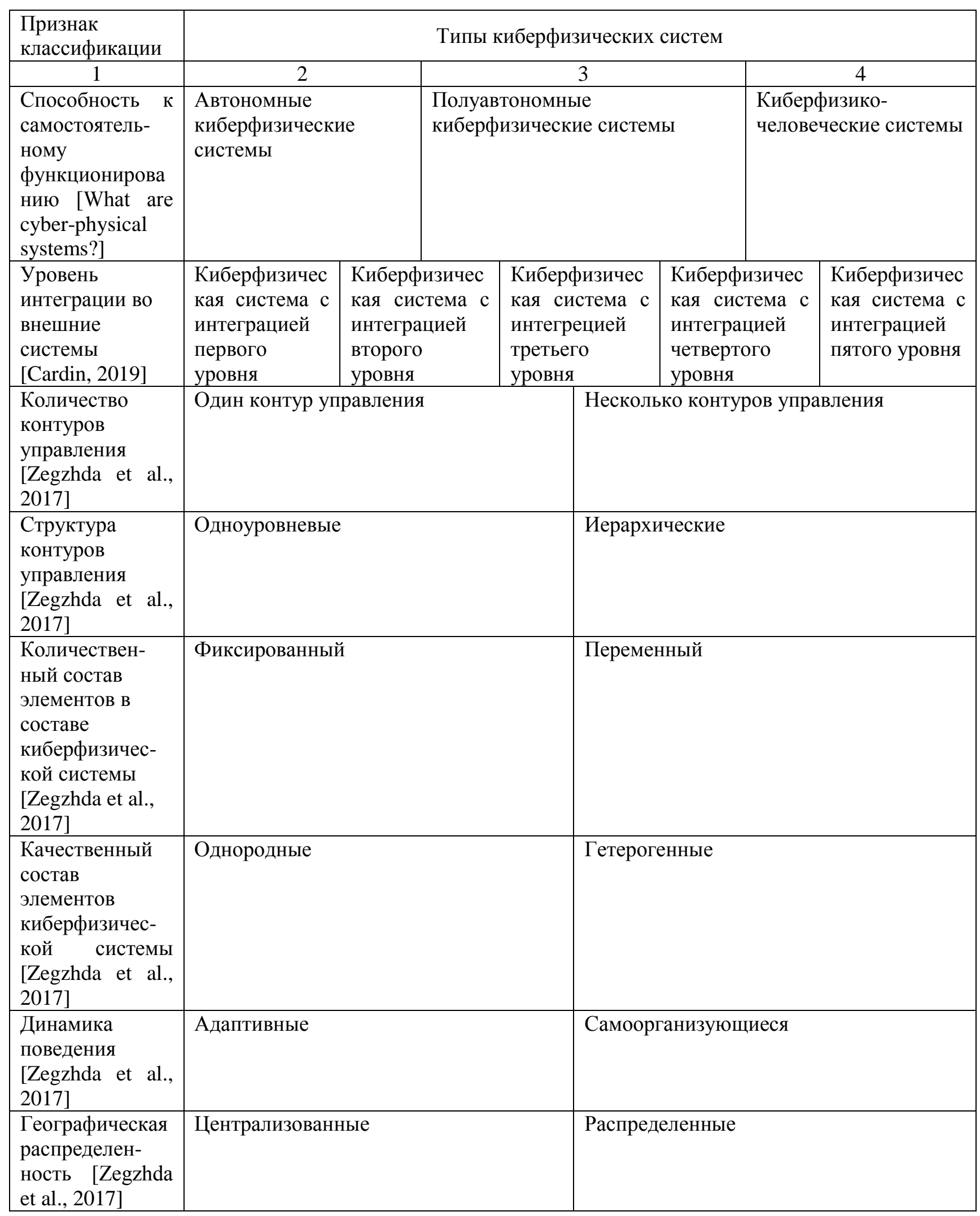


Окончание табл. 1

\begin{tabular}{|c|c|c|c|c|c|c|c|c|}
\hline 1 & \multicolumn{4}{|c|}{2} & \multicolumn{4}{|c|}{3} \\
\hline $\begin{array}{l}\text { Связь с внешней } \\
\text { средой [Zegzhda } \\
\text { et al., 2017] }\end{array}$ & \multicolumn{3}{|l|}{ Открытые } & \multicolumn{5}{|c|}{ Закрытые } \\
\hline $\begin{array}{l}\text { Принадлежность } \\
\text { к отрасли } \\
\text { [Громаков, } \\
\text { Сидорова, 2021] }\end{array}$ & $\begin{array}{l}\text { Промышлен- } \\
\text { ность }\end{array}$ & $\begin{array}{l}\text { Сельское } \\
\text { хозяйство }\end{array}$ & Меди & цина & Энергетика & Тран & ппорт & $\begin{array}{l}\text { Образование } \\
\text { и т.д. }\end{array}$ \\
\hline $\begin{array}{l}\text { Масштаб } \\
\text { [Громаков, } \\
\text { Сидорова, 2021] }\end{array}$ & \multicolumn{3}{|l|}{$\begin{array}{l}\text { Отдельные } \\
\text { устройства } \\
\text { (беспилотный } \\
\text { транспорт) }\end{array}$} & \multicolumn{3}{|c|}{$\begin{array}{l}\text { «Умные» } \text { фабрики } \\
\text { (SM) }\end{array}$} & \multicolumn{2}{|c|}{$\begin{array}{l}\text { Умные } \quad \text { сети } \\
\text { (Интернет вещей) }\end{array}$} \\
\hline $\begin{array}{l}\text { Специфика } \\
\text { инжиниринга }\end{array}$ & $\begin{array}{l}\text { Новое } \\
\text { программный I }\end{array}$ & $\begin{array}{l}\text { удование } \\
\text { дукт } \\
\end{array}$ & & $\begin{array}{l}\text { Име } \\
\text { про }\end{array}$ & $\begin{array}{lr}щ е е с я ~ & \text { обб } \\
\text { aммный прод }\end{array}$ & $\begin{array}{l}\text { рудс } \\
\text { укт }\end{array}$ & & новый \\
\hline
\end{tabular}

В зависимости от способности к самостоятельному функционированию Роберто Сабатини выделяет следующие типы CPS [What are cyber-physical systems?]:

1) Автономные киберфизические системы способны самостоятельно принимать решения и не зависеть от человека.

2) Полуавтономные киберфизические системы работают независимо только в определенных условиях. Например, оператор задает траекторию движения дрона, что позволит ему обогнуть препятствия, а значит, самостоятельно совершать полет, без ручного управления.

3) Киберфизико-человеческие системы - это системы, требующие при необходимости вмешательства человека.

Одна из особенностей CPS - подключение к сети. В зависимости от уровня интеграции вовне различают следующие типы [Cardin, 2019]:

1) Киберфизическая система с интеграцией первого уровня использует готовые данные датчиков сети.

2) Киберфизическая система с интеграцией второго уровня использует данные датчиков, обрабатывает и агрегирует их.

3) Киберфизическая система с интеграцией третьего уровня распознает другие киберфизические системы и взаимодействует с ними для обогащения собственной обработки данных.

4) Киберфизическая система с интеграцией четвертого уровня обрабатывает данные сети и использует их для диагностики своего состояния.

5) Киберфизическая система с интеграцией пятого уровня способна самостоятельно адаптироваться к изменениям, регулировать свои параметры в автономном режиме.

$\mathrm{B}$ соответствии со спецификой инжиниринга классификация CPS приобретает следующий вид [Zegzhda et al., 2017]:

1) Киберфизические системы, при создании которых использовалось новое оборудование и программный продукт;

2) Киберфизические системы, созданные на базе уже имеющегося оборудования и нового программного продукта.

Киберфизические системы могут иметь один или несколько контуров управления. Каждый из них представляет собой совокупность датчиков, управляющих компьютеров и исполнительных органов. Структура контуров управления может быть одноуровневой или иерархической [Zegzhda et al., 2017]. В зависимости от этого выделяют две группы CPS.

Еще одним принципом классификации киберфизических систем является количественный состав элементов в их составе [Zegzhda et al., 2017]. Он может быть постоянным или переменным. 
В зависимости от качественного состава элементов выделяют [Zegzhda et al., 2017]:

1) однородные киберфизические системы, т. е. системы, состоящие из одинаковых по своим характеристикам и свойствам элементов;

2) гетерогенные киберфизические системы, т. е. системы, состоящие из разнородных компонентов, отличающихся своими свойствами и характеристиками.

CPS отличаются динамикой поведения. Так, адаптивные киберфизические системы подстраиваются под среду, способны прогнозировать внешние изменения. В то же время самоорганизующиеся киберфизические системы совершенствуют собственную организацию как под воздействием внешних факторов, так и в случае стабильной работы [Zegzhda et al., 2017].

В соответствии с географической распределенностью киберфизические системы бывают централизованными (системы, расположенные в рамках одного физического объекта, например, на одном предприятии, в одном здании) и распределенными (системы, расположенные на нескольких связанных между собой объектах) [Zegzhda et al., 2017].

Одним из классификационных признаков стало наличие с внешней средой. Для открытых киберфизических систем необходим Интернет, а для закрытых достаточно внутренней сети [Zegzhda et al., 2017].

Киберфизические системы могут быть классифицированы в зависимости от отрасли, в которой они функционируют: в промышленности, сельском хозяйстве, медицине, энергетике и т. д. Масштаб может варьироваться от отдельных устройств (беспилотный транспорт) и «умных» домов до «умных» фабрик (SM) и умных сетей (Интернет вещей) [Громаков, Сидорова, 2021].

Предлагаем новый классификационный признак - специфика инжиниринга. В соответствии с ним в первую группу включены киберфизические системы, при создании которых использовалось новое оборудование и программный продукт, а во вторую созданные на базе уже имеющегося оборудования и нового программного продукта.

Представленная классификация свидетельствует о многообразии киберфизических систем и об их сложной структуре, предполагающей объединение программного и аппаратного обеспечения под управлением высококвалифицированных кадров. Степень участия человека в работе CPS при этом отличается в каждом конкретном случае. При анализе киберфизических цифровых проектов следует учесть этот фактор.

Внедрение киберфизических систем в промышленности является трудоемким и затратным процессом. В связи с этим требуется предварительная оценка эффективности реализации такого проекта. Наиболее известным методом анализа инвестиционных проектов является Модель ООН по промышленному развитию UNIDO [Behrens, Hawranek, 1991]. Подход считается универсальным, а в его основе лежит модель «затраты-выгоды». UNIDO позволяет оценить количественно отдачу от инвестиционных вложений, выявить срок их окупаемости, используя такие показатели, как, например, NPV и IRR. Большинство современных методик, базируется на UNIDO. По мнению исследователей, существенным недостатком подхода является то, что он упускает из вида риски инвестирования. Кроме того, UNIDO ориентирован на долгосрочную перспективу, а в условиях цифровизации сроки реализации проектов сжатые и требуют оперативного мониторинга.

Отечественным аналогом этого международного метода являются «Методические рекомендации по оценке эффективности инвестиционных проектов» (утв. Минэкономики РФ, Минфином РФ, Госстроем РФ 21.06.1999 N ВК 477) [Методические рекомендации по оценке эффективности инвестиционных проектов, 1999]. Суть этого подхода заключается в применении финансовых показателей UNIDO. Их расчет осуществляется с помощью прогнозных значений денежного потока. В итоге формируется система из таких показателей, как чистый дисконтированный доход, срок окупаемости проекта, внутренняя норма доходности и индекс доходности/прибыльности. Методика считается универсальной, поэтому применима для цифровых инвестиционных проектов, однако, на наш взгляд, как и 
UNIDO, она, во-первых, упускает из виду риски, а во-вторых, не учитывает специфику работы киберфизической системы с точки зрения оценки эффективности работы аппаратного обеспечения. В результате, в ряде случаев, опираясь исключительно на оценку дисконтированных денежных потоков, можно отказаться от проектов, направленных на рост эффективности использования оборудования.

В части анализа нефизической составляющей CPS возможен укрупненный анализ рентабельности затрат и экспертная оценка качественных характеристик цифрового продукта. Кроме того, программное обеспечение неразрывно связано с аппаратным в составе киберфизической системы. Эффективность работы оборудования определяется одновременно со связанным с ним эффектом от цифрового решения. В связи с этим считаем целесообразным сосредоточиться на анализе физической части CPS.

При реализации цифрового инвестиционного проекта она является наиболее затратной, и, что примечательно, финансовые вложения требуются не только для приобретения непосредственно самого оборудования, но и для подготовки инженерной инфраструктуры и производственной площадки. В связи с этим нужно четко понимать, какого результата позволит достичь модернизация. Причем в случае с аппаратной составляющей киберфизической системы в промышленности анализ должен быть комплексным и учитывать не только абсолютные значения стоимости или количества выпущенной продукции, но и ее качество, эффективность использования временных и трудовых ресурсов.

Анализ существующих на данный момент методик оценки эффективности работы оборудования показал, что выявленным нами требованиям в наибольшей степени соответствует показатель OEE (Overall Equipment Effectiveness). В соответствии с ГОСТ Р ИСО 22400-2-2019 ОЕЕ - это индекс, характеризующий «эксплуатационную готовность рабочего элемента, его эффективность и коэффициент готовой продукции, объединенные в один показатель» [ГОСТ Р ИСО 22400-2-2019 Системы промышленной автоматизации и интеграция, 2019]. Единица изменения показателя - проценты. В классическом варианте формула расчета ОЕЕ выглядит следующим образом:

$$
\text { OEE }=\text { Д } * \Pi * \mathrm{~K},
$$

где ОЕЕ - индекс общей эффективности использования оборудования, \%;

Д - доступность или эксплуатационная готовность оборудования - это объем потерь из-за простоя. Она характеризует пропорцию времени между временем фактической работы оборудования и временем его загрузки и рассчитывается как соотношение фактического времени работы к времени загрузки оборудования в часах [ГОСТ Р ИСО 22400-2-2019 Системы промышленной автоматизации и интеграция. 2019];

П - производительность или показатель эффективности деятельности - это коэффициент эффективности производственной деятельности, связывающий чистое рабочее время и общее время работы оборудования. Он позволяет выяснить, присутствует ли потеря производительности производства. Коэффициент эффективности производственной деятельности рассчитывается как отношение чистого рабочего времени к общему времени работы в часах;

К - качество, которое оцифровывается через коэффициент готовой продукции. Он показывает долю качественной продукции, которую удалось произвести, и рассчитывается как отношение качественной продукции к общему объему продукции в натуральных единицах или стоимостной оценке.

Таким образом, в соответствии с классической формулой ОЕЕ анализирует различные характеристики работы оборудования, используя метрики простоев, изменения скорости работы и изменение качества результата.

Существует еще один вариант расчета OEE: 


$$
\mathrm{OEE}=\frac{\mathrm{AT}}{\mathrm{TPR} * \mathrm{SOP}}[\text { Расчет общей эффективности оборудования (OEЕ)], }
$$

где ОЕЕ - индекс общей эффективности использования оборудования, \%;

AT (Actual Throughput), фактическая пропускная способность - объем качественной продукции;

TPR (Theoretical Processing Rate), теоретическая скорость обработки - скорость обработки в узком месте системы при наличии идеальных условий;

SOT (Scheduled Operating Time), расчетное время производства - количество, запланированное системой, времени для работы, включая производство и обслуживание.

Представляется, что первый подход к оценке эффективности работы оборудования через показатель ОЕЕ более приемлем, поскольку в такой записи более четко просматриваются ключевые факторы, определяющие эффективность и, следовательно, появляется возможность проведения факторного анализа, по результатам которого могут быть приняты соответствующие управленческие решения. Мы проанализировали и систематизировали факторы, которые могут отрицательно влиять на каждый из показателей в составе ОЕЕ (табл. 2).

Остановимся подробнее на каждом из выявленных параметров.

«Отказ» оборудования представляет собой потерю доступности к работе на этом оборудовании, то есть незапланированный простой в связи с поломкой, отсутствием квалифицированных кадров, нехваткой оборотных средств. Его причиной также может стать отсутствие части оборудования на каком-либо этапе на линии при поточном производстве, что делает невозможным выпуск готовой продукции.

Компоненты OEE

Components of OEE

\begin{tabular}{|l|l|}
\hline \multicolumn{1}{|c|}{ Составляющая ОЕЕ } & \multicolumn{1}{|c|}{ Факторы, влияющие на составляющие ОЕЕ } \\
\hline \multirow{2}{*}{ Доступности } & «Отказ» оборудования \\
\cline { 2 - 2 } & Настройка и регулировка \\
\cline { 2 - 2 } & Остановки по вине оператора оборудования \\
\hline \multirow{2}{*}{ Производительность } & Холостой ход и непродолжительные остановки \\
\cline { 2 - 2 } & Пониженная скорость работы \\
\hline \multirow{2}{*}{ Качество } & Технологические дефекты (брак) \\
\cline { 2 - 2 } & Снижение количества качественной продукции \\
\hline
\end{tabular}

Важно отличать «отказ» оборудования (потеря доступности) от замедления производственного процесса (потери производительности). Для этого должно быть регламентировано время остановок оборудования, например, час, по истечении которого простой считается «отказом» оборудования по определенным причинам.

Настройка и регулировка занимает в ряде случае достаточно продолжительный период времени, в течение которого оборудование не задействовано в производственном процессе в связи с осуществлением переналадки, прогрева, планового технического обслуживания.

Холостой ход и непродолжительные остановки - параметр, являющийся причиной потери производительности. Такая проблема возникает вследствие замятия материала, его неправильной подачи, неправильных настроек оборудования. Такие остановки длятся не более 5 минут и не требуют привлечения персонала, осуществляющего ремонт оборудования. Оператор самостоятельно устраняет возникающие проблемы. Холостой ход и непродолжительные остановки рассматриваются как потеря производительности, часто имеют хронический характер. 
Пониженная скорость работы также характеризует производительность. Она приводит к тому, что процесс на производстве занимает больше времени, чем теоретически возможно в соответствии с мощностью оборудования. Такая ситуация возникает вследствие износа оборудования, использования некачественных материалов, низкой квалификацией оператора, работающего на станке.

Технологические дефекты (брак) включают в себя некачественную продукцию, а также продукцию, не соответствующую требованиям и подлежащую переработке на стабильном производстве. Этот фактор учитывается, так как ОЕЕ при анализе эффективности работы оборудования берет в расчет только продукт, изначально произведенный как качественный. Причинами дефектов, как правило, становятся некорректные настройки оборудования, ошибки оператора, его неправильное обращение с техникой или истечение срока годности сырья, что актуально, например, для фармацевтической продукции.

Измерение снижения объема качественной продукции актуально в период с момента запуска оборудования до достижения момента его стабильной работы. Фактор, как и технологические дефекты, включает в себя некачественную продукцию, а также продукцию, не соответствующую требованиям и подлежащую переработке. Отличие заключается в том, что его контролируют после установки или переналадки оборудования.

C описанными факторами сталкиваются все промышленные предприятия, осуществляющие внедрение нового оборудования или киберфизической системы, то есть интегирированного комплекса оборудования и цифрового решения. Воздействуя на каждый из них, возможно контролировать итоговую эффективность работы.

Однако приведенная формула ОЕЕ, в состав которой входит несколько показателей работы оборудования, несмотря на свой комплексный характер, не позволяет в полной мере учесть специфику таких цифровых инвестиционных проектов как CPS. Киберфизические системы, сочетая в своей структуре физический компонент (датчики, измерительную технику, биологический объект - человека) и программный продукт, обладают рядом особенностей. Во-первых, они самостоятельно осуществляют мониторинг своего состояния, что позволяет своевременно проводить плановые ремонты и избегать простоев оборудования. Данное свойство учитывается в доступности. Во-вторых, киберфизические системы позволяют повысить качество изделий, что находит отражение в коэффициенте готовой продукции. В-третьих, основной целью модернизации в промышленности является повышение производительности. Такой эффект внедрение киберфизической системы также находит отражение в формуле расчета OEЕ в показателе эффективности деятельности.

Наконец, CPS характеризуются высокой степенью автономности, но при этом одним из наиболее важных физических компонентов в их структуре остается человек. Приведенная классификация киберфизических систем по степени участия оператора в работе системы свидетельствует о том, что его роль существенна. Человек выполняет ряд функций, обеспечивающих бесперебойность производственных процессов: фиксирует сигнал о потребности в ремонте, принимает решение о переналадке оборудования.

Таким образом, мы убеждены, что при анализе цифрового киберфизического проекта должна быть учтена эффективность работы оператора, иначе результат оценки будет некорректен. Кроме того, это такой подход соответствует современным результатам исследований, которые среди причин неудач цифровых программ признают весьма существенную роль такой причины как низкая, либо недостаточная квалификация персонала, связанного с цифровым оборудованием. В этом случае показатели эффективности оборудования позволяют получить высокие значения дисконтированных показателей эффективности проекта, а человеческий фактор не дает возможности проявить эти показатели на практике.

Для решения этой проблемы мы модифицировали формулу расчета ОЕЕ, включив в нее показатель оценки эффективности деятельности человека в киберфизической системе: 


$$
\mathbf{O E E}=\frac{\mathbf{t}_{\mathbf{p r}}+\mathbf{o}+\mathbf{n}}{\mathbf{t}_{\text {plan }}} * \frac{\mathbf{t}_{\mathbf{i}} * \mathbf{Q}_{1}}{\mathbf{t}_{\mathbf{p r}}+\mathbf{t}_{\mathbf{0}}} * \frac{\mathbf{Q}_{2}}{\mathbf{Q}_{1}}
$$

где ОЕЕ - индекс общей эффективности использования оборудования, \%;

$\mathrm{t}_{\mathrm{pr}}$ - общий период производственного процесса, в течение которого оборудование функционировало без нарушений, в часах;

о - период времени, в течение которого оборудование простаивало из-за его отказа, в часах;

$\mathrm{n}$ - период времени, который заняла настройка и регулировка работы оборудования, в часах;

$\mathrm{t}_{\text {plan }}$ - период времени, в течение которого по нормативу должно было работать оборудование, в часах;

$\mathrm{t}_{\mathrm{i}}$ - идеальное время цикла, то есть период времени, в течение которого должно быть выпущено максимально возможное количество продукции, в часах;

$\mathrm{Q}_{1}$ - объем продукции, выпущенный за период производственного процесса, в течение которого оборудование функционировало без нарушений, в единицах;

$\mathrm{t}_{0}$ - период времени, в течение которого зафиксирован холостой ход оборудования, непродолжительные остановки, в часах;

$\mathrm{Q}_{2}$ - количество деталей, соответствующих стандартам качества, которые установлены для продукции, в часах.

Допустим, что общий период производственного процесса, в течение которого оборудование функционировало без нарушений, равен идеальному времени цикла (период времени, в течение которого должно быть выпущено максимально возможное количество продукции, в часах), так как цель - производить максимальное количество продукции в единицу времени. Вследствие этого формула приобретает следующий вид:

$$
\mathbf{O E E}=\frac{\mathbf{t}_{\mathbf{p r}}+\mathbf{o}+\mathbf{n}}{\mathbf{t}_{\mathrm{pr}}+\mathbf{t}_{\mathbf{0}}} * \mathbf{Q}_{1},
$$

Добавим показатель оценки эффективности деятельности оператора в киберфизической системе, в результате чего классическая версия формулы в укрупненном виде будет выглядеть следующим образом:

$$
\mathbf{O E E}=Д * \Pi * \mathrm{~K} * \mathbf{P},
$$

где ОЕЕ - индекс общей эффективности использования оборудования, \%;

Д - доступность или эксплуатационная готовность.

П - производительность или показатель эффективности деятельности.

К - качество (коэффициент готовой продукции).

P -эффективность деятельности оператора.

Эффективность деятельности оператора в киберфизической системе будем рассчитывать по следующей формуле:

$$
\mathbf{P}=\frac{\mathbf{Q}_{\mathrm{t}}}{\mathbf{V}_{\mathrm{t}} * \mathbf{t}_{\mathbf{p}}}
$$

где $\mathrm{Q}_{\mathrm{t}}$ - количество продукции, произведенной за период времени работы оператора на оборудовании, единиц;

$\mathrm{V}_{\mathrm{t}}$ - средняя скорость работы оператора в течение общего периода времени работы, единиц в час;

$\mathrm{t}_{\mathrm{p}}$ - общая продолжительность периода времени, в течение которого оператор работал на оборудовании, в часах.

С учетом приведенной выше формулы, расчет показателя ОЕЕ в развернутом виде будет выглядеть следующим образом:

$$
O E E=\frac{t_{p r}+o+n}{t_{p r}+t_{0}} * Q_{1} * \frac{Q_{t}}{V_{t} * t_{p}}
$$

Предложенная формула позволяет решить проблему учета влияния человеческого фактора на результат работы киберфизической системы. В ней учтены 4 группы факторов, 
характеризующих одновременно эффективность работы физико-биологической составляющей (оборудование, оператор) и программной составляющей - цифрового продукта в киберфизической системе.

Теоретически диапазон значений ОЕЕ варьируется от 0 до $100 \%$. Нормативное значение показателя, свидетельствующее о высоком уровне организации производства, зависит от отрасли. Например, ОЕЕ для отрасли производства фасованных потребительских товаров составляет 40-50\%, мировые лидеры с производством циклического типа добиваются эффективности $80 \%$, а при производстве непрерывного типа - даже $90 \%$ [Обобщённая эффективность оборудования (OEЕ) для совершенствования производства фасованных потребительских товаров]. Существует мнение, что оптимальные значения для отельных компонентов приняты следующие: доступность - $90 \%$, производительность - $95 \%$, качество - 99,9\% (рис. 1). При этих значениях ОЕЕ составит 85,4 \%. По данным мировой практики, пороговое значение эффективности лидеров производства - 85 \%, и именно к этому значению стремятся промышленные компании.

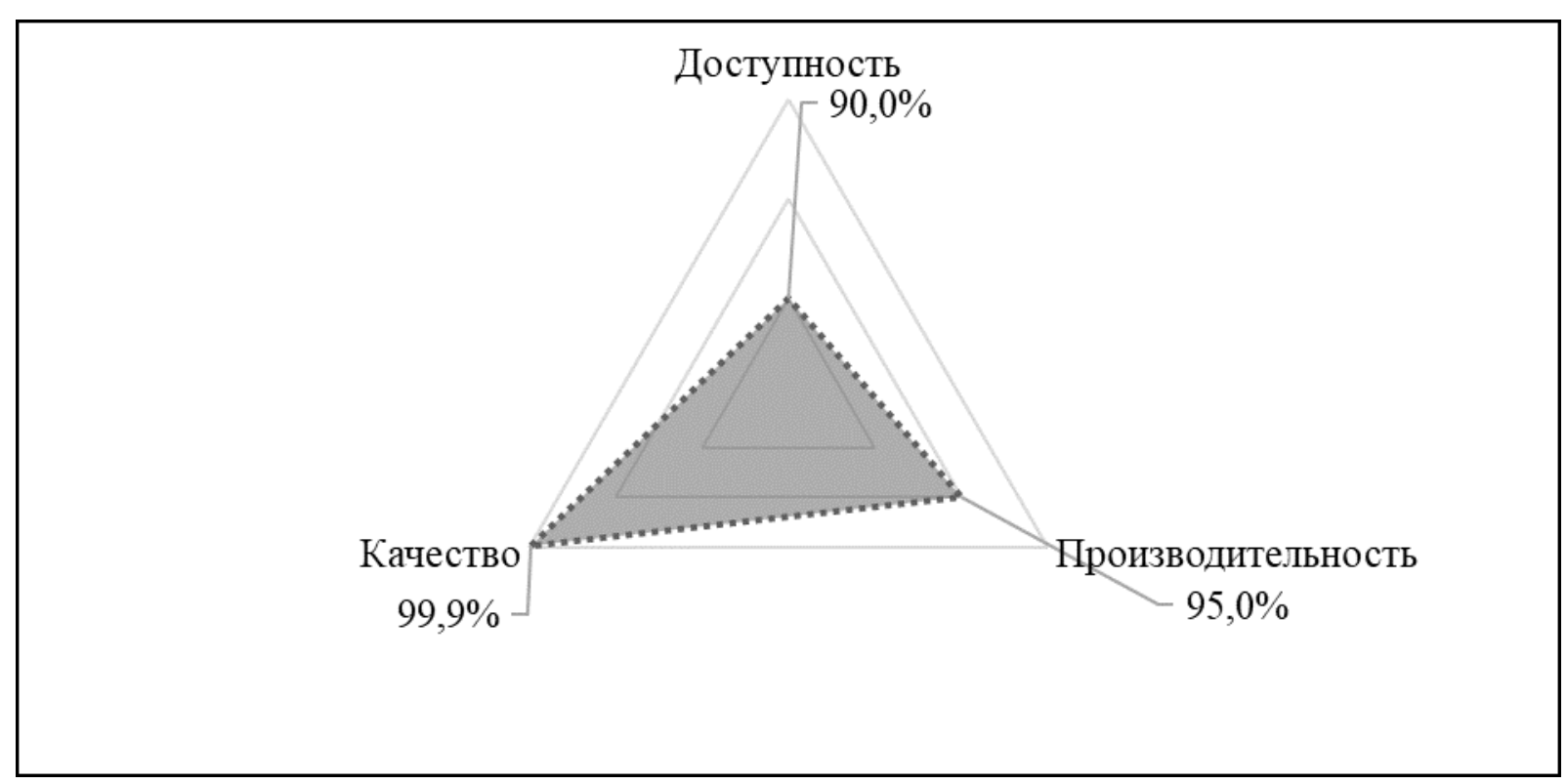

Рис. 1. Показатели компонентов ОЕЕ, \%

Fig. 1. Indicators of OEE components, \%

Важно отметить, что компоненты в составе OEE неоднородны [Шопин и др.], что, в том числе, доказывают разные оптимальные значения каждой составляющей, приведенные выше. Доступность и производительность показывают потери, возникновение которых обусловлено недопроизводством. Его причинами становятся потери времени в связи с простоями, настройкой и доработкой, остановками по вине оператора, а также пониженная скорость работы и холостой ход. Выпуск продукции в меньшем объеме по сравнению с тем, который был запланирован, с точки зрения доступности обусловлен тем, что оборудование не работало совсем, а с точки зрения производительности - работало непродуктивно. Такие потери можно считать виртуальными, так как они измеряются соотношением реального и теоретически возможного объема.

Компонент, характеризующий в составе OEE качество, напротив, показывает фактические потери. Бракованная продукция уже выпущена и от нее придется либо избавиться совсем, либо подвергнуть переработке, что влечет за собой дополнительные затраты.

Эффективность деятельности человека в киберфизической системе показывает потери, которые возникают в связи с пониженной скоростью выполнения функций 
оператором и его низкой квалификацией, в связи с чем возникают непредвиденные остановки и замедляется общий темп работы.

Данные статистики, приведенные выше, доказывают, что вклад каждого из компонентов ОЕЕ неравномерен и несет разную нагрузку. На данном этапе исследования нам известны средние значения каждой составляющей: доступность - $90 \%$, производительность - $95 \%$, качество - 99,9\% [Мартиросян, 2017]. В предложенном нами варианте расчета ОЕЕ установим нормативное значение для нового компонента эффективности работы оператора. Проведенный анализ по подбору параметра позволил определить, что при заданных значениях доступности, производительности и качества эффективность работы оператора должна составлять 99,98 \% (рис. 2).

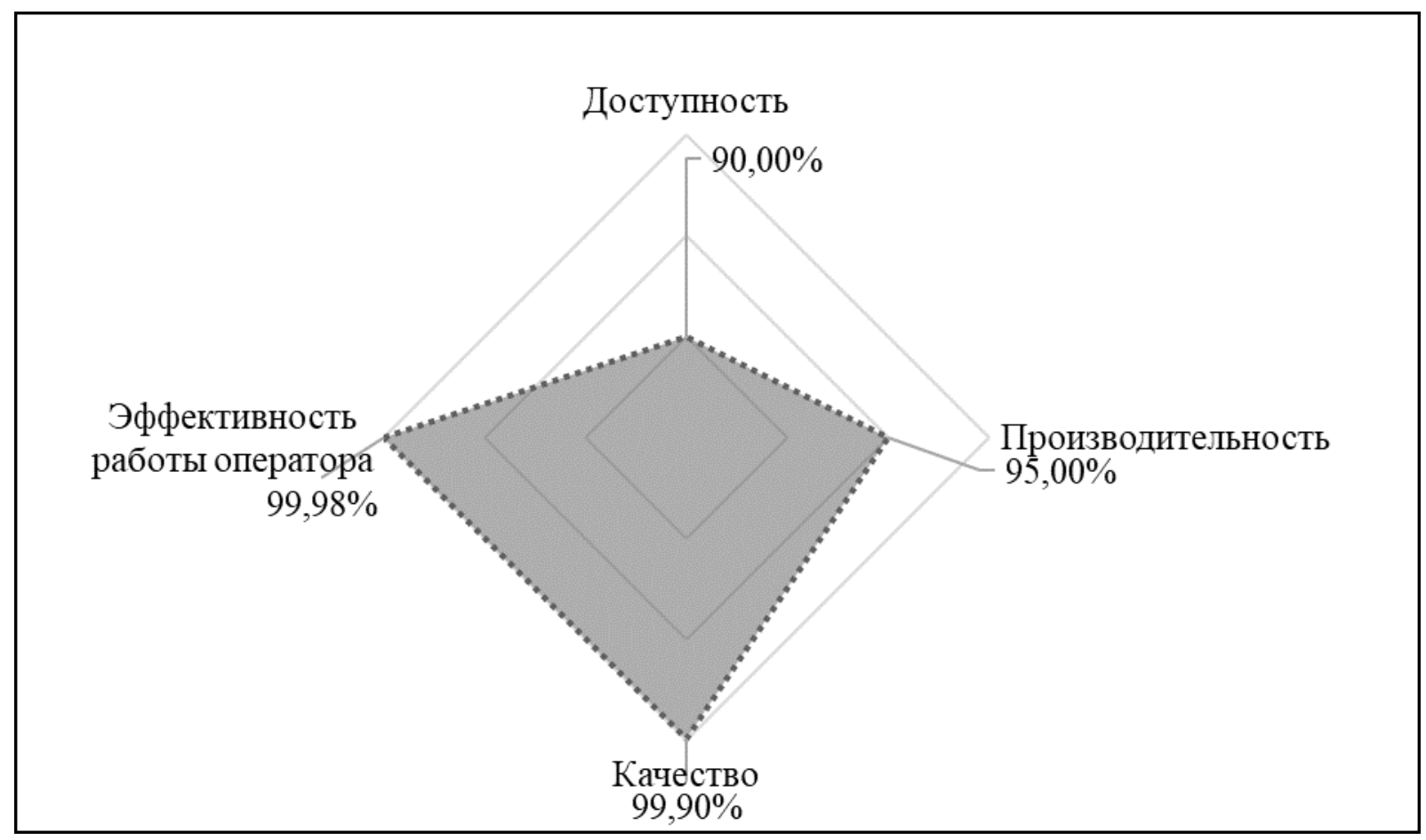

Рис. 2. Показатели компонентов ОЕЕ с учетом эффективности работы оператора, \% Fig. 2. Indicators of OEE components considering the operator's efficiency, \%

Другими словами, квалификация оператора должна быть максимальна, так, чтобы его ошибки не привели к снижению эффективности киберфизической системы и, соответственно, отказу от проекта ее внедрения.

Как уже отмечалось, в зависимости от отрасли и специфики производства нормативное значение каждой составляющей ОЕЕ может отличаться от приведенного нами (см. рис. 1, 2). Однако итоговый показатель должен стремиться к 100 \%. Чтобы добиться этого, необходимо управлять каждой составляющей формулы: доступностью, производительностью, качеством и эффективностью работы оператора. В связи с неоднородностью компонентов для каждого из них допускается свое пороговое значение (см. рис. 2), однако крайне важно не допускать существенного превышения одного показателя над другим, иначе общая эффективность системы резко снизится.

Для осуществления факторного анализа, прежде всего, следует определить значение показателя ОЕЕ и его отдельных составляющих (рис. 3).

При значении компонента «доступность» ниже нормативного (90 \%) необходимо выяснить, за счет чего возникают потери времени. Возможные причины простоев представлены на рисунке 3 (см. рис. 3). Для их сокращения можно сформулировать следующий ряд рекомендаций. 
1. Регулярные проверки оборудования и проведение плановых ремонтов для предупреждения «отказов» оборудования.

2. Применение технологии бережливого производства SMED (eng. Single-Minute Exchange of Die) с целью минимизации временных потерь на настройку и регулировку [Быстрая переналадка для рабочих, 2009].

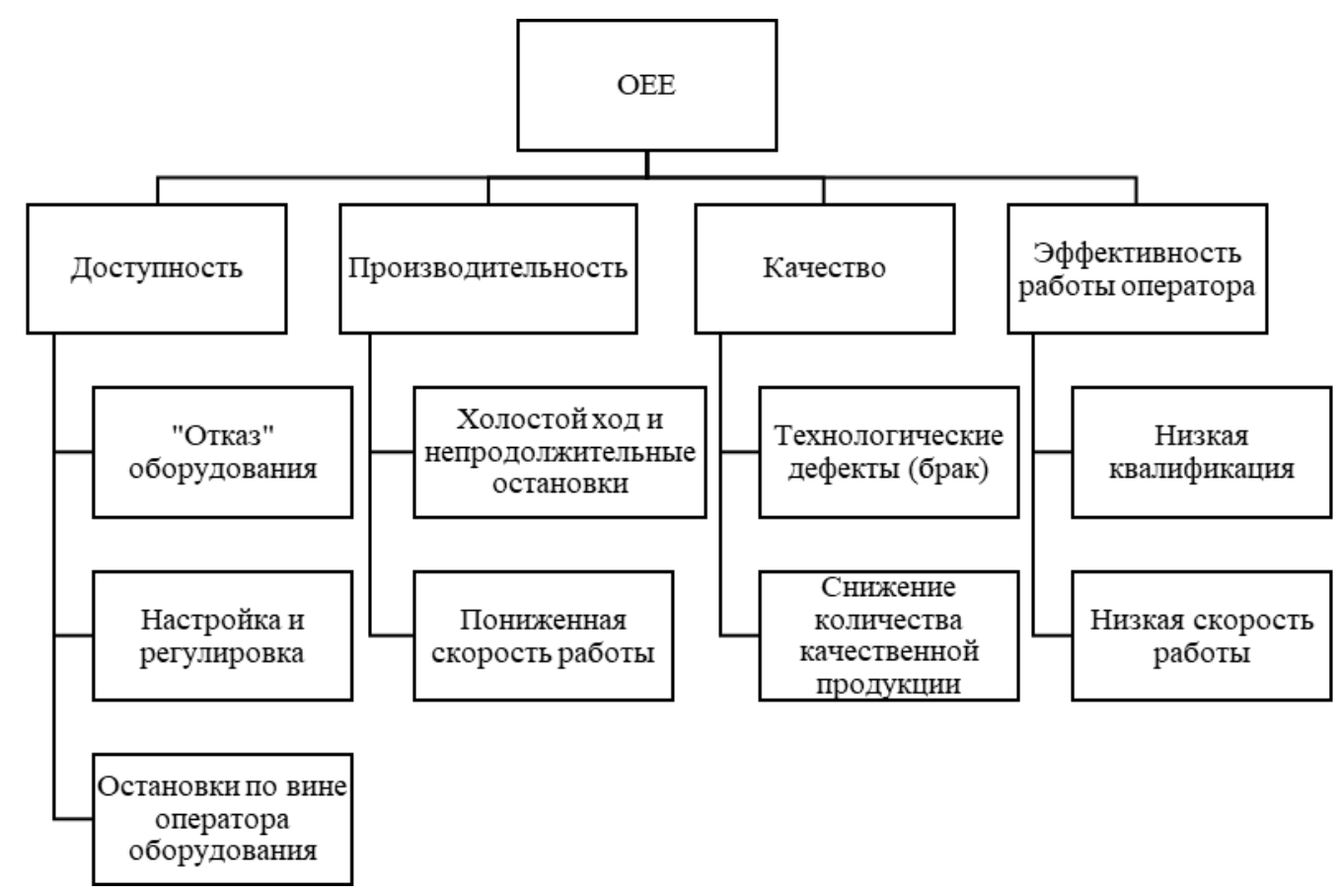

Рис. 3. Факторы управления показателем ОЕЕ

Fig. 3. Factors driving the OEE score

SMED - это организация переналадки с минимальными временными затратами. Она включает ряд мероприятий: описание текущего процесса настройки и регулировки и выявление в нем «узких» мест, формирование команды для реализации новой технологии, обучение персонала. Кроме того, следует разделить операции по переналадке на два группы [Быстрая переналадка для рабочих, 2009].

Первая - это обязательные внутренние операции - требуют обязательной остановки оборудования.

В целях их оптимизации рекомендуется использовать передвижные установки для инструментов и стопорные механизмы для быстрого изменения положения зажимов, стандартизировать используемые для переналадки детали. Такие незначительные на первый взгляд мероприятия позволяют в совокупности сэкономить значительное количество времени.

Вторая - это обязательные внешние операции - не требуют обязательной остановки оборудования.

Чтобы они не отнимали много времени, следует внедрить принципы визуального контроля и использовать чек-листы, провести организацию рабочей зоны по принципу $5 \mathrm{~S}$.

3. Минимизация остановок по вине оператора за счет правильного подбора и обучения персонала, проведения хронометража рабочего времени.

В качестве основных факторов, посредством которых можно влиять на производительность как компонент OEE, были выделены: холостой ход и непродолжительные остановки, пониженная скорость работы. В первом случае причиной простоя становится неправильная подача материала, некорректная настройка, очистка оборудования. Конкретные мероприятия для воздействия на производительность будут зависеть от влияющих на нее факторов. Например, при неправильной подаче материала 
следует контролировать его качество и проверить оборудование на предмет поломки. Пониженная скорость, влияющая на производительность, обусловлена износом механизмов, условиями среды, не позволяющими работать с максимальной скоростью, качеством используемых материалов. В данном случае также нужно регулярно выполнять плановый мониторинг, следить за качеством материалов.

На качество влияют технологические дефекты (брак) и снижение объема качественной продукции. Важно отметить, что первый фактор характеризует уже устоявшееся производство. Брак в этом случае возникает по технологическим причинам (неправильная настройка оборудования) или, например, при использовании неподходящего сырья. Снижение объема качественной продукции, как правило, наблюдается после переналадки. Причиной такой проблемы становится некорректная настройка, неоптимальные регулировки. Управление качеством в данном случае предполагает внедрение технологии бережливого производства, одним из этапов которой является строгий контроль результатов переналадки.

Новым компонентом ОЕЕ - эффективностью работы оператора - следует управлять через повышение квалификации персонала и повышением скорости его работы. Для этого необходимо регулярно проводить обучение, контролировать квалификацию посредством сдачи экзаменов на знание особенностей работы оборудования. Повышение скорости работы оператора возможно с помощью хронометража, с учетом данных которого оптимизируется время операций [Чухрова, 2021].

Таким образом, достижение высокого показателя ОЕЕ осуществляется посредством планомерного и регулярного управления факторами, влияющими на доступность, производительность, качество и человеческий фактор.

\section{Заключение}

В условиях становления концепции «Индустрия 4.0» особую актуальность приобретает искусство инжиниринга, заключающееся в способности объединять физические и цифровые составляющие, в результате чего появляются киберфизические системы, представляющие собой особый вид цифровых инвестиционных проектов. Проведенный анализ позволил среди всего многообразия CPS выделить несколько групп таких проектов и классифицировать их по ряду признаков, в том числе принципиально новых, таких как масштаб, специфики отрасли и особенностей инжиниринга. На основе представленной типологии киберфизических систем выявлена их специфика, которую следует учитывать при анализе эффективности соответствующих проектов. Этому требованию, на наш взгляд, соответствует модифицированный метод оценки ОЕЕ.

Для комплексного анализа эффективности киберфизической системы, помимо предложенного метода, целесообразно использовать и классические показатели эффективности, в частности, методику UNIDO. Однако OEE - это наиболее адекватный специфике CPS подход, непосредственно оценивающий физические и биологические компоненты, которые при этом неразрывно связаны и находятся под влиянием цифрового решения в структуре киберфизической системы.

\section{Список источников}

1. Архангельская Е., Осипов И., Волкова О., Вайнер Н., Травникова Е. 40 лет с Apple: сорок фактов о самой дорогой компании мира. URL: https://www.rbc.ru/technology_and_media/ 01/04/2016/56fd6cf99a79477e0c2cf5b4 (дата обращения: 20 октября 2021).

2. ГОСТ Р ИСО 22400-2-2019 Системы промышленной автоматизации и интеграция. Ключевые технико-экономические показатели (KPIs) для управления производственными операциями. Часть 2. Определения и описания. 2019. URL: https://allgosts.ru/13/020/ gost_r_iso_224002-2019 (дата обращения: 20 октября 2021). 
3. Громаков Е.И., Сидорова А. А. 2021. Современные технологии. Киберфизические системы: учебное пособие. Томский политехнический университет. Томск: Изд-во Томского политехнического университета, 166 c. URL: https://portal.tpu.ru/SHARED/s/SIDOROVA/ Yheba/Tab8/\%D0\%A1\%D0\% BE\%D0\%B2\%D1\%80\%D0\%B5\%D0\%BC\%D0\%B5\%D0\%BD\%D0\%BD\%D1\%8B\%D0\%B5\%20\%D1\%8 $2 \% \mathrm{D} 0 \% \mathrm{~B} 5 \% \mathrm{D} 1 \% 85 \% \mathrm{D} 0 \% \mathrm{BD} \% \mathrm{D} 0 \% \mathrm{BE} \% \mathrm{D} 0 \% \mathrm{BB} \% \mathrm{D} 0 \% \mathrm{BE} \% \mathrm{D} 0 \% \mathrm{~B} 3 \% \mathrm{D} 0 \% \mathrm{~B} 8 \% \mathrm{D} 0 \% \mathrm{~B} 8$.pdf $\quad$ (дата обращения: 12 ноября 2021 г.).

4. Клейменова Л. 2021. РБК Тренды. Что такое индустрия 4.0 и что нужно о ней знать. URL: https://trends.rbc.ru/trends/industry/5e 740c5b9a79470c22dd13e7 (дата обращения: 12 ноября 2021 г.).

5. Круглов С. Эволюция телефона: от Александра Белла до наших дней. URL: https://tass.ru/spec/phone (дата обращения: 20 октября 2021).

6. Методические рекомендации по оценке эффективности инвестиционных проектов (утв. Минэкономики РФ, Минфином РФ, Госстроем РФ 21.06.1999 № BK 477). 1999. URL: http://www.consultant.ru/document/cons doc LAW 28224/ (дата обращения: 15 октября 2021 г.).

7. Обобщённая эффективность оборудования (OEЕ) для совершенствования производства фасованных потребительских товаров. URL: https://technolink.spb.ru/wp-content/uploads/2018/07/ equipment-efficiency.pdf (дата обращения: 15 октября 2021 г.).

8. Расчет общей эффективности оборудования (OEE). URL: https://bpi-group.com.ua/blog/raschetobshhej-effektivnosti-oborudovaniya-oee/ (дата обращения: 15 октября 2021 г.).

9. Руководство по цифровой трансформации производственных предприятий. 2019. URL: http://assets.fea.ru/uploads/nticenter/112019/Rukovodstvo_po_cifrovizacii_proizvodstvennyh_predpriyatij.p df (дата обращения: 15 октября 2021 г.).

10. Рыжков А. 2009. Быстрая переналадка для рабочих. М.: Институт комплексных стратегических исследований, 112 c. URL: https://biznesbooks.com/components/com_jshopping/ files/demo_products/productivity-press-bystraya-perenaladka-dlya-rabochikh.pdf (дата обращения: 20 октября 2021).

11. Чухрова Н. Хронометраж рабочего времени. URL: https://www.gd.ru/articles/12066hronometraj-rabochego-vremeni (дата обращения: 12 ноября 2021 г.).

12. Шопин А.Г., Занин И.В., Спиридонов С.В. OEE/DTM - Золушка в семействе MESрешений. URL: https://studylib.ru/doc/2491358/oee-dtm-\%E2\%80\%93-zolushka-v-semejstve-mes-reshenij (дата обращения: 20 ноября 2021 г.).

13. Behrens W., Hawranek P.M. 1991. Manual for the preparation of industrial feasibility studies.

14. Cardin O. Classification of cyber-physical production systems applications: proposition of an analysis framework. 2019. URL: https://arxiv.org/ftp/arxiv/papers/1811/1811.03122.pdf (дата обращения: 20 ноября 2021 г.).

15. Cyber-Phisical System. Driving force for innovation in mobility, healthy, energy and production. ACATECH 2011.

16. Lee E.A., Seshia S.A. 2017. Introduction to embedded systems: A cyber-physical systems approach. London: MIT Press. URL: https://ptolemy.berkeley.edu/books/leeseshia/releases/ LeeSeshia_DigitalV2_2.pdf (дата обращения: 20 ноября 2021 г.).

17. What are cyber-physical systems? URL: https://www.rmit.edu.au/news/c4de/what-are-cyberphysical-systems (дата обращения: 20 ноября 2021 г.).

\section{Список литературы}

1. Мартиросян А.Т. 2017. Показатель эффективности обслуживания оборудования в системе ключевых показателей производительности. Интернет-журнал «НАУКОВЕДЕНИЕ», 9(6). URL: https://naukovedenie.ru/PDF/164EVN617.pdf (дата обращения: 20 ноября 2021 г.).

2. Трошин А., Пискайкин М. 2021. Инжиниринг и «Индустрия 4.0». Разбор терминологии. Компоненты и технологии, 9. URL: https://www.mosep.ru/local/images/moselproj/kit-ing.pdf (дата обращения: 12 ноября 2021 г.).

3. Zegzhda D.P., Poltavtseva M.A., Lavrova D.S. 2017. Systematization and security assessment of cyber-physical systems. Automatic control and computer sciences, 51(8): 835-843.

\section{References}

1. Martirosyan A.T. 2017. Efficiency indicator of equipment maintenance in the system of key performance indicators. Online magazine «Science», 9(6). URL: https://naukovedenie.ru/PDF/ 164EVN617.pdf (in Russian). 
2. Troshin A., Piskaykin M. 2021. Engineering and Industry 4.0. Analysis of terminology. Components and technologies, 9. URL: https://www.mosep.ru/local/images/moselproj/kit-ing.pdf (in Russian).

3. Zegzhda D.P., Poltavtseva M.A., Lavrova D.S. 2017. Systematization and security assessment of cyber-physical systems. Automatic control and computer sciences, 51(8): 835-843.

Конфликт интересов: о потенциальном конфликте интересов не сообщалось.

Conflict of interest: no potential conflict of interest related to this article was reported.

\section{ИНФОРМАЦИЯ ОБ АВТОРАХ}

Любименко Дарья Анатольевна, аспирант кафедры экономики и финансов ЮжноУральского государственного университета (НИУ), г. Челябинск, Россия

Вайсман Елена Давидовна, доктор экономических наук, профессор, профессор кафедры экономики и финансов ЮжноУральского государственного университета (НИУ), г. Челябинск, Россия

\section{INFORMATION ABOUT THE AUTHORS}

Darya A. Lyubimenko, Postgraduate Student of the Department of Economics and Finance, South Ural State University (National Research University), Chelyabinsk, Russia

Elena D. Vaisman, Doctor of Economics, Professor, Professor of the Department of Economics and Finance, South Ural State University (National Research University), Chelyabinsk, Russia 